\title{
Tecnologia: novas abordagens, conceitos, dimensões e gestão
}

\author{
José Carlos Teixeira da Silva \\ Dr. Eng. Produção / Escola Politécnica - USP \\ Prof. Dr. Depto. Eng. de Produção - Unesp - Bauru - SP \\ Email: jcteixe@feb.unesp.br
}

\begin{abstract}
Resumo
Este trabalho discute conceitos e dimensões do termo "tecnologia", no campo da gestão da tecnologia em empresas de manufatura. Considerando a tecnologia embutida em produtos/processos, e a capabilidade tecnológica nas organizações, foi possível desdobrar o "conteúdo da tecnologia", criando os conceitos de "macrotecnologia" e "microtecnologia". Enquanto a macrotecnologia é referente ao conceito sistêmico dentro da organização, a microtecnologia envolve a tecnologia embutida em um produto/processo. Esses conceitos permitem análises estratégicas e operacionais no campo da gestão da tecnologia nas organizações. Uma pesquisa de campo preliminar indicou limitações das organizações com relação aos componentes da microtecnologia e da macrotecnologia.
\end{abstract}

\section{Palavras-chave}

Tecnologia, gestão da tecnologia, desenvolvimento do produto.

\section{Technology: news approaches, concepts, dimensions and management}

\begin{abstract}
This paper discusses technology concepts and dimensions, in the field of technology management at the manufacture sector, with respect to product and process. Considering the embedded technology in products/ process, and the technology capability in the organizations, was possible the deployment of the term "technology", creating the concepts of "macrotechnology" and "microtechnology". While the macrotechnology is referent to the systemic concept of technology inside the organization, the microtechnology is referent to the technology inside the product/process. These concepts allow to analyze strategic and operational aspects of technology management. A preliminary field research has detected limited capability in organizations with respect the components of microtecnology and macrotechnology.
\end{abstract}

\section{Key words}

Technology, technology management, product development. 


\section{INTRODUC̣ÃO E OBJETIVOS}

A gestão da tecnologia ("technology management" ou "management of technology") é um campo recente de abordagem multidisciplinar, dentro das dimensões atuais de competitividade das empresas na economia globalizada. Pode-se dizer que os primeiros passos ocorreram a partir da segunda metade da década de 1980 nos Estados Unidos da América - EUA, envolvendo governo, empresas e universidades. Essa abordagem é decorrente de movimentos no campo da teoria organizacional das empresas.

Um histórico do sistema produtivo de maior escala, com praticamente dois séculos de maturação, indica diferentes fases quanto à forma organizacional da empresa, da produção e do trabalho, bem como nas relações com o mercado e o meio ambiente, e sobretudo como são desenvolvidas e utilizadas as tecnologias de produto e de processo de produção. Na última metade do século XVIII a máquina a vapor constituiu uma inovação tecnológica importante, pois permitiu mecanizar o sistema produtivo, aumentando a escala de produção, e agilizar os meios de transporte, com as ferrovias e a navegação, permitindo a expansão dos mercados e o escoamento da produção industrial, agrícola e agroindustrial. Constata-se o surgimento das indústrias ferroviárias e navais, com as novas tecnologias de acionamento, e uma série de equipamentos e componentes até então nunca utilizados pelos métodos anteriores de transporte. A expansão da produção agroindustrial, ao longo das ferrovias, quer seja no velho mundo ou no novo mundo, abriu fronteiras agrícolas nunca antes exploradas, permitindo o escoamento do excedente da produção dos países para outras regiões do globo.

O movimento da qualidade empreendido pelas empresas japonesas, a partir da década de 1960, influenciou o setor industrial de todos os países, no campo da abordagem organizacional da empresa, da produção e do trabalho, pela sua diferenciação da teoria da administração científica taylorista do início do século XX. A adaptação das empresas ocidentais àquela forma organizacional alternativa passou a se constituir um paradigma, tendo em vista as diferenças entre as culturas ocidental e japonesa. Após quase uma década de adaptações, no início da década de 1990 constatase certo equilíbrio do nível de produtividade e de qualidade entre as chamadas "empresas de classe mundial", segundo a classificação de Merli (1994). Essa situação induziu a um direcionamento adicional, a partir da década de 1990, para outra dimensão de competitividade relacionada à inovação em produtos, em processos e em tecnologia. A inovação em produtos / tecnologia passa a ser então função de aspectos internos e externos à empresa, dentro de um meio ambiente externo dinâmico e de transformações. Internos, no que se refere a uma forma organizacional que permita comunicação contínua entre todos os níveis da organização, incluindo os aspectos de planejamento estratégico, de aprendizagem, de geração de conhecimentos, e de competência. Externos, quanto ao seu relacionamento com o mercado e meio ambiente, aí incluídos os consumidores, fornecedores, concorrentes, e a análise constante da evolução tecnológica e do mercado. As empresas que ainda não se adaptaram a essa nova forma de gestão têm uma dupla missão, dentro dessa complexidade de reformulação organizacional, envolvendo simultaneamente a gestão da qualidade e a gestão da tecnologia, como fatores de competitividade e sobrevivência no mercado.

\section{A gestão da tecnologia ("technology management" - ou "management of technology"] é um campo recente de abordagem multidisciplinar, dentro das dimensões atuais de competitividade das empresas} na economia globalizada.

As pequenas e médias empresas - PMEs podem ser consideradas como fundamentais para a atividade econômica e representam um instrumento de geração de empregos e de inovação, quando bem gerenciadas. Entretanto, as oportunidades de negócios para essas empresas, em um mercado globalizado, estão limitadas por uma variedade de fatores, entre eles, as dificuldades de acesso à informação e integração naquele mercado, além da limitada capabilidade de gestão da tecnologia para geração de novas tecnologias de produtos/processos que resultem em produção comercial com sucesso. Essas empresas têm motivado a preocupação governamental em países desenvolvidos e em desenvolvimento, no que refere ao fator emprego dentro do processo de globalização, diretamente relacionado à manutenção e expansão de seus mercados, e também função dos níveis de competitividade nos campos da gestão da qualidade e da gestão da tecnologia de produtos/processos.

O presente trabalho discute aspectos relacionados aos conceitos da palavra "tecnologia", bem como o desenvolvimento de novas abordagens e dimensões, dentro da configuração da gestão da tecnologia em empresas de manufatura, 
independentemente de sua dimensão, envolvendo principalmente o campo das tecnologias de produto/processo e a capabilidade tecnológica da organização. Uma pesquisa de campo preliminar também foi conduzida, através de entrevistas, em pequenas e médias empresas tradicionais do setor de manufaturados, visando detectar o nível de conhecimento dessas organizações nesse campo.

\section{CONCEITOS E NOVAS ABORDAGENS DA DIMENSÃO TECNOLOGIA}

A utilização da palavra "tecnologia" vem sendo ampliada para muitas áreas do conhecimento, alterando muitas vezes seu significado e distanciando-se da conceituação tradicional. Assim, seria oportuno caracterizar o significado da palavra "tecnologia" que será utilizado neste trabalho. Os conceitos envolvendo desenvolvimento de novos produtos, da mesma forma como abordaram Wheelwright; Clark (1992) e Utterback (1994), devem ser diferenciados do conceito de inovação tecnológica. Vamos considerar o desenvolvimento do produto como o processo de transformação de idéias, dados de mercado e tecnologias, em novos conhecimentos para geração de produtos e serviços em produção comercial. A palavra tecnologia nesse contexto poderá significar tecnologias já conhecidas ou novas tecnologias, uma vez que um novo produto poderá ou não incorporar novas tecnologias. O conceito de tecnologia poderá ter maior ou menor abrangência, dependendo do foco em análise. Segundo Longo (1984), "tecnologia é o conjunto de gica em pequenas e médias empresas é mais ampla, ou seja, "tecnologia é o conjunto de conhecimentos necessários para se conceber, produzir e distribuir bens e serviços de forma competitiva", o que engloba todos os conhecimentos relacionados às atividades da empresa.

Portanto, o conceito de tecnologia utilizado neste trabalho envolve todos esses conceitos, embora na área específica de desenvolvimento do produto possa parecer, à primeira vista, que esteja incorporada somente a conceituação de Blaumer (1964) apud Fleury (1978) anteriormente referenciada. Os dados de mercado embutidos nos conceitos envolvem todos os aspectos externos à empresa, que interferem em sua área de atuação, incluindo fornecedores, concorrentes, produtos, clientes, etc., e as idéias significam o ponto de partida do desenvolvimento, sendo uma dimensão dependente de vários fatores para sua viabilização.

Tradicionalmente, a tecnologia utilizada por uma organização, dependendo das condições do meio ambiente, poderá ser desenvolvida internamente ou absorvida externamente através de contratos de pesquisa, licenciamento, participação minoritária ou majoritária em outras empresas, e "joint-ventures". Quando do desenvolvimento interno com sucesso comercial, tanto para o produto, como para o processo, a organização adquire competência naquela tecnologia. De acordo com Steensma (1996), essa capacitação tecnológica está diretamente associada a aspectos organizacionais que envolvem comunicação entre as pessoas e aprendizagem, e indica que "aprendizagem organizacional é o processo de intermediação entre a interação colaborativa e a aquisição da competência técnica", em nível da organização. A aprendizagem individual, segundo Aegyris; Schom (1978) apud Steensma (1996), “é necessária mas não suficiente para a organização de aprendizagem (organizational learning)", e como as-

conhecimentos científicos ou empíricos empregados na produção e comercialização de bens e serviços". A conceituação de Blaumer (1964) apud Fleury (1978) se concentra mais na fabricação, ou seja, "se refere ao conjunto de objetos físicos e operações técnicas (mecanizadas ou manuais) empregadas na transformação de produtos em uma indústria”, similar à proposição de Abetti (1989) apud Steensma(1996), que define tecnologia como "um corpo de conhecimentos, ferramentas e técnicas, derivados da ciência e da experiência prática, que é usado no desenvolvimento, projeto, produção, e aplicação de produtos, processos, sistemas e serviços". Já a conceituação utilizada por Kruglianskas (1996), quando analisa a gestão da inovação tecnoló- sinala Meyers (1990) apud Steensma (1996), "parece que o aprendizado da organização é diferente da soma dos conhecimentos dos indivíduos que a compõem". Portanto, quando do desenvolvimento interno de uma tecnologia ou novo produto em uma empresa, existe um ambiente de conformação que leva a organização a adquirir capabilidade para esse tipo de atividade, ou seja, uma competência da organização no campo daquela tecnologia ou novo produto, extrapolando o conhecimento ou a competência individual das pessoas que fazem parte daquele meio ambiente. Essa competência, que denominaremos de "capabilidade tecnológica", é uma característica implícita daquela organização, tipicamente intangível, naquele escopo de tecnologia e naquele momen- 
to, sendo praticamente impossível sua transferência global para outras organizações, pois é função daquele meio ambiente. Esse aspecto da gestão da tecnologia ou desenvolvimento de novos produtos é fundamental para a empresa, porém ainda pouco estudado, uma vez que ele não pode ser generalizado, ou melhor, sua caracterização é complexa e inerente à própria organização. Por isso é que a "transferência de tecnologia" entre organizações constitui um aspecto complexo, pois a organização receptora não tem o mesmo ambiente da organização "doadora" daquela tecnologia. Essa complexidade varia em função da característica da tecnologia a ser absorvi$\mathrm{da}$, sendo menor para tecnologias similares à existente na organização, e maior para mudanças no escopo da tecnologia. Em sua análise sobre aquisição de competência técnica através da colaboração inter-organizacional, sob o ponto de vista de aprendizado da organização receptora, Steensma (1996) relaciona os diversos níveis de colaboração (contrato de pesquisa, licenciamento, investimento minoritário, joint-ventures, aquisição do controle) com o nível de aprendizado existente (simples, adaptativo, transição, criativo), e conclui que "quanto maior o nível de aprendizado existente em uma empresa, maior sua capacidade de assumir um compromisso inter-organizacional de absorção de tecnologia". Nesse contexto, quando da pouca capacitação da empresa para a absorção, a universidade poderia intermediar esse processo na geração de capacitação, indo além dos contratos de pesquisa tradicionais, resguardados os aspectos de sigilo e ética envolvidos.

A gestão da tecnologia na empresa poderá ter seu espectro ampliado em função do conceito adotado para a palavra "tecnologia". Uma conceituação ampliada poderá facilitar a visualização do estágio tecnológico de uma determinada organização em um determinado instante, independentemente de sua dimensão, apesar das dificuldades existentes no campo das pequenas e médias empresas com a problemática da transposição de teorias organizacionais desenvolvidas em grandes empresas, de forma similar ao que ocorreu no campo da gestão da qualidade.

Tecnologia é um tópico extenso que pode significar muitas coisas para as pessoas. Martino (1983) cita que o Webster's Seventh Collegiate Dictionary define "tecnologia" como "the totality of the means employed to provide objects necessary for human sustenance and comfort". Uma versão mais generalizada do conceito de tecnologia poderia ser : "tecnologia é um sistema através do qual a sociedade satisfaz as necessidades e desejos de seus membros". Esse sistema contém equipamentos, programas, pessoas, processos, organização, e finalidade de propósito. Nesse contexto, um produto é o artefato da tecnologia, que pode ser um equipamento, programa, processo, ou sistema, o qual por sua vez pode ser parte do meio ou sistema contendo outra tecnologia. 
Ainda recentemente, a indústria de manufatura americana definia seu desempenho com base em tecnologias disponíveis e naquilo que o consumidor queria. Ocasionalmente aumentava qualidade com aumento de desempenho. De acordo com Dertouzoz; Lester; Solow (1989), o aumento de custos com o aumento aleatório da qualidade, ao invés de baixos custos e alta qualidade, levou a indústria americana a perder vantagem competitiva. Em contraste, dentro do conceito sistêmico, o Japão projetou sua "nova forma organizacional", visando alto valor agregado, e incorporando "alta qualidade" e "baixo preço", incorporando métodos para ouvir o consumidor e desenvolver novos produtos, através de conceitos de desenvolvimento de produto e processo integrados, do desdobramento da função qualidade, custos, confiabilidade, além do desenvolvimento de novos mercados. Assim, somente dentro dessa nova metodologia (sistêmica organizacional) é possível perceber o que as indústrias japonesas realmente fizeram para ter vantagem competitiva.

Dentro de uma visão sistêmica, o termo "transferência de tecnologia" dificilmente se aplica, pois significaria transferência de todos os aspectos do sistema, de acordo com a Figura 1. É claro que a transferência de somente uma parte do sistema não transfere tecnologia.

É evidente que esse conceito sistêmico de tecnologia, o qual designaremos de "macrotecnologia", constitui a base para a gestão e uso das tecnologias propriamente ditas, ou seja, aquelas que designaremos de "microtecnologias", que envolvem as tecnologias principais e as tecnologias complementares, como veremos posteriormente. Análises preliminares de alguns aspectos relacionados a esse campo foram conduzidas anteriormente em Silva (2001), Silva (2002a) e Silva (2002b). Esses conceitos se inserem no contexto de produtos/processos, tanto novos como existentes, pois a maioria dos desenvolvimentos utiliza tecnologias já existentes. Para abordagem com novas tecnologias, os componentes da "macrotecnologia" assinalados anteriormente são necessários, mas não suficientes, para ter sucesso no empreendimento, pois além de alto grau de "criatividade" o mercado deve "puxar" em direção à inovação.

As tecnologias de produto/processo utilizadas por uma organização são dependentes das condições do meio ambiente, mas devem ser competitivas no mercado em que atua. Preponderantemente devem ser desenvolvidas dentro da organização. No campo das PMEs tradicionais, entretanto, poderá existir limitação para esse processo, pois ele é dependente da existência de capacitação tecnológica da empresa, e esta, de acordo com Steensma (1996), como já citamos anteriormente, "está diretamente associada a aspectos organizacionais que envolvem a comunicação entre as pessoas e a aprendizagem". Adicionalmente, a capabilidade tecnológica incorpora também uma "habilidade" na gestão e operacionalização de um planejamento estratégico

\section{Figura 1: Conceitos Embutidos na Macrotecnologia.}

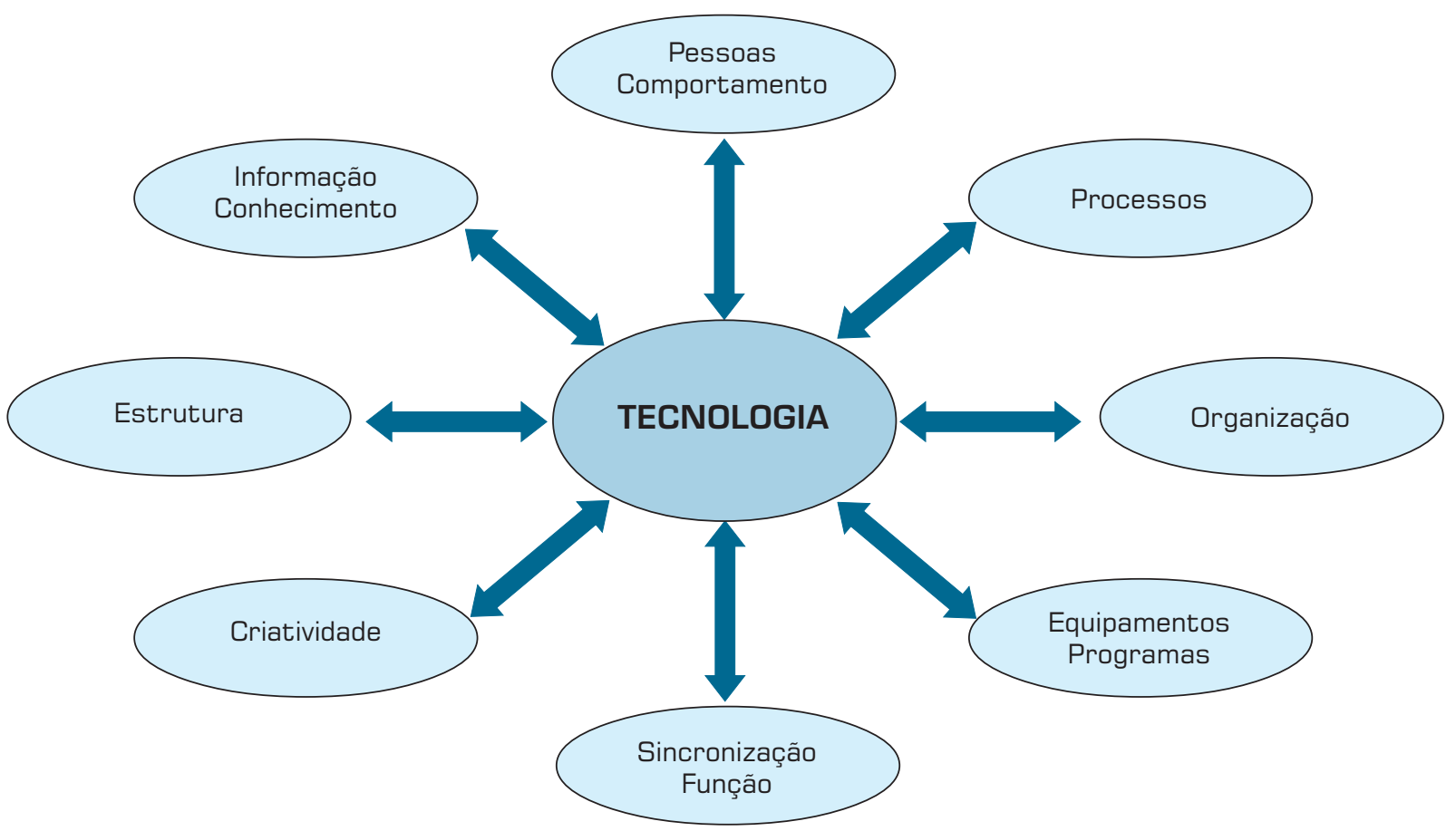


de tecnologia em direção às melhorias incrementais contínuas e novos produtos/processos, como assinalaram Silva; Plonski (1996) e Silva (2002b) quando analisam a inovação tecnológica na empresa. Para uma economia globalizada o "conceito de macrotecnologia" indica que o termo "transferência de tecnologia" é impraticável, devido à impossibilidade de transferência de todas as pessoas, conhecimentos e facilidades, do sistema de tecnologia de uma organização para outra.

No contexto atual da economia globalizada, uma empresa que não desenvolve suas próprias tecnologias de produto e de processo não é competitiva, embora possa estar temporariamente competitiva, dependendo do setor em que atua. Essa vulnerabilidade tecnológica poderá ser analisada em função das características das tecnologias de produto e de processo utilizadas pela organização em um determinado instante, de acordo com a Figura 2.

Com a orientação adotada na Figura 2, no campo de tecnologias já conhecidas ou novas, podemos analisar o estágio de vulnerabilidade quanto ao produto e ao processo, em relação ao domínio dessas tecnologias, ou seja, públicas ou restritas. Quando utilizamos o termo "domínio público" significa uma tecnologia de domínio público, ou seja, qualquer concorrente poderá dispor dessa tecnologia a qualquer momento, pois os direitos de propriedade industrial já foram universalizados, ou são de baixo valor agregado. Quando utilizamos o termo "domínio restrito" significa uma tecnologia que não é de domínio público, podendo ocorrer duas possibilidades: uma relacionada à existência de propriedade industrial (própria ou licenciada); e a outra de propriedade da empresa, ainda não licenciada. Podemos ter uma empresa com reduzido conteúdo tecnológico embutido em um produto, porém com razoável conteúdo tecnológico no processo, ou seja, vulnerabilidade baixa. Quando a empresa gera um produto com conteúdo tecnológico, mas com processo de domínio público, a vulnerabilidade pode ser considerada média. No caso de tecnologias de produto e processo com reduzidos conteúdos tecnológicos, a vulnerabilidade pode ser considerada alta. Muitas empresas desconhecem a legislação pertinente à propriedade industrial, e expõem suas tecnologias aos concorrentes. Evidentemente, as tecnologias embutidas em um produto estão expostas no mercado, o que não ocorre com as tecnologias de processo de produção, que são internas à empresa. Nesse ponto seria interessante colocar que existe distinção quando falamos de tecnologia de processo e de tecnologia de fabricação. A

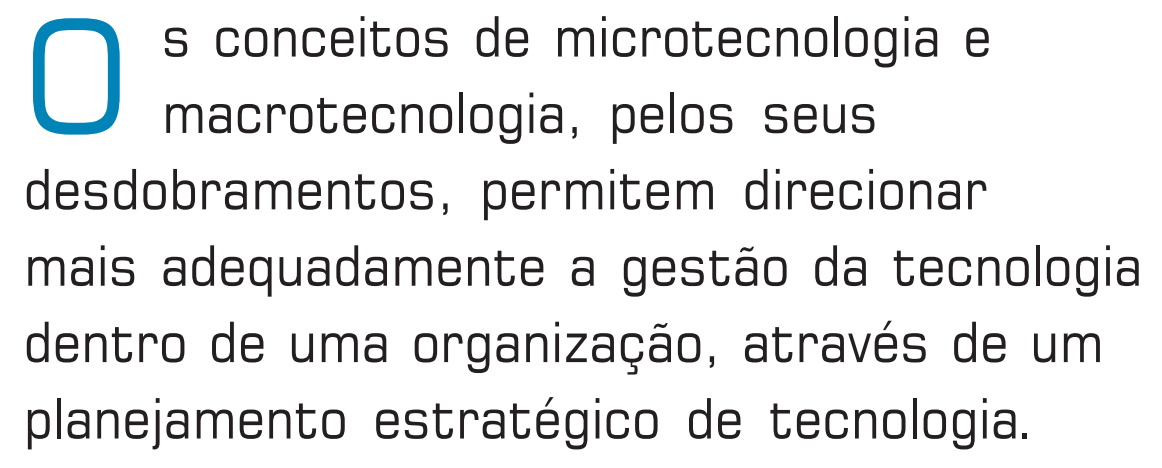

tecnologia de processo é definida pela empresa quando do desenvolvimento de um novo produto, e a tecnologia de fabricação está relacionada às etapas do processo, ou seja, função dos equipamentos de produção adquiridos de terceiros, como por exemplo de fabricantes de bens de capital. As tecnologias embutidas em um bem de capital são bastante relevantes no contexto da gestão da tecnologia em uma empresa, incluindo o contexto daquela que adquire o equipamento, que passa a ser dependente da tecnologia utilizada pela empresa que produz o "bem de capital".

No caso de novas tecnologias, ainda de acordo com a Figura 2, a vulnerabilidade tecnológica poderá ser média e baixa, dependendo se o domínio for restrito ou público, pois a nova tecnologia poderá ser de produto ou processo, ou ambas. Evidentemente, a vulnerabilidade tecnológica é uma dimensão temporal, pois estamos analisando um determinado instante histórico da empresa (como uma fotografia), isto significando que o termo "não vulnerabilidade" não pode ser utilizado, pois nenhuma tecnologia se enquadra nesse contexto ao longo do tempo, pela difusão e transformação dos conhecimentos.

Figura 2: Vulnerabilidade das Tecnologias na Empresa.

\begin{tabular}{|l|c|c|c|}
\hline & Produto & Processo & Vulnerabilidade \\
\hline $\begin{array}{l}\text { Tecnologia } \\
\text { Conhecida }\end{array}$ & $\begin{array}{c}\text { Domínio Público } \\
\text { Domínio Restrito } \\
\text { Domínio Restrito }\end{array}$ & $\begin{array}{c}\text { Domínio Público } \\
\text { Domínio Público } \\
\text { Domínio Restrito }\end{array}$ & $\begin{array}{c}\text { Alta } \\
\text { Média } \\
\text { Baixa }\end{array}$ \\
\hline $\begin{array}{l}\text { Nova } \\
\text { Tecnologia }\end{array}$ & $\begin{array}{c}\text { Domínio Restrito } \\
\text { Domínio Restrito }\end{array}$ & $\begin{array}{c}\text { Domínio Público } \\
\text { Domínio Restrito }\end{array}$ & Média \\
\hline
\end{tabular}


Quando falamos em tecnologias de produto e processo, quer sejam conhecidas ou novas, na realidade estamos nos referindo a um conjunto de tecnologias inter-relacionadas dentro do contexto de um produto ou de um processo, ao qual designaremos daqui para a frente pela terminologia "microtecnologia". Tanto com relação ao produto, como para o processo, podemos esquematizar, de modo similar, os relacionamentos, de acordo com a Figura 3.

Vamos introduzir nesse esquema alguns conceitos para descrição dos conjuntos. O termo "Tecnologia PrincipalTP" (que em linguagem inglesa poderia ser "Core Technology-CT") se refere à tecnologia principal do produto ou do processo, ou seja, aquela que distingue aquele produto ou processo de outros produtos e processos, dentro das finalidades específicas de utilização. O termo "Tecnologia Complementar-TC" (que analogamente poderia ser "Boundary Technology-BT") se refere às tecnologias, não obrigatoriamente desenvolvidas pela empresa geradora do produto ou processo, que colaboram para a operacionalização da tecnologia principal, como, por exemplo, partes e componentes.

Com relação aos produtos, o valor agregado do conteúdo tecnológico poderá ser maior ou menor, como, por exemplo, um veículo automotor ou um balde de plástico, respectivamente. Enquanto que no veículo a CT e a BT são importantes, no balde elas praticamente não se caracterizam. Nos veículos automotores a CT é concentrada no motor a combustão interna, e as BTs em outras partes como transmissão, suspensão, freios, refrigeração, geração de corrente elétrica, aerodinâmica etc. Na maioria dos automóveis a CT, que significou uma inovação tecnológica importante no século XIX, praticamente não sofreu alterações, enquanto houve grande evolução das BTs. Com relação à CT em um automóvel, atualmente já existem alternativas ao motor a combustão interna, como, por exemplo, o motor elétrico a bateria, energia solar, célula de combustível, significando assim alterações na TP ou CT. Podemos afirmar que a maioria das melhorias incrementais em produtos ocorre nas "boundaries technologies" e não nas "core technologies". Um exemplo de alterações da CT de um produto já existente é o caso do toca-disco de vinil convencional e o tocadisco laser $\mathrm{CD}$, onde a tecnologia principal foi substituída. Outro exemplo de tecnologias distintas pode ser constatado nas máquinas de lavar roupas com eixos verticais e horizontais, onde as tecnologias principais são distintas. Essa alteração da tecnologia principal, e algumas das complementares, faz com que aquela de eixo horizontal utilize em parte a gravidade para o processo de lavagem, com resultados surpreendentes na redução do consumo de energia, água, sabão-em-pó, e aumento da eficiência de lavagem. Esses

Figura 3: Microtecnologia: Conjunto de Tecnologias de um Produto ou Processo.

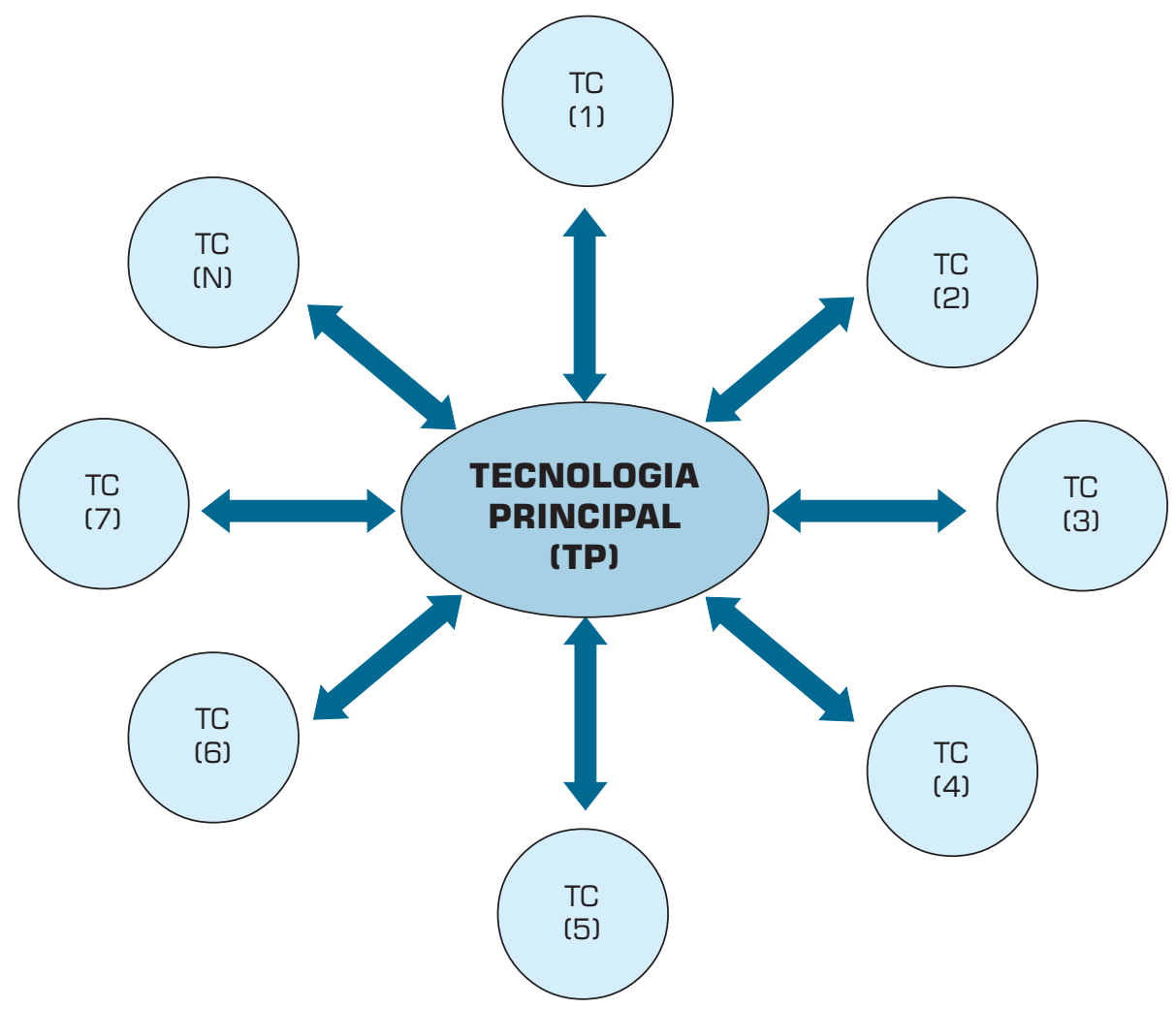


dados são conhecidos, pois toda lavagem industrial utiliza equipamentos com eixos horizontais.

De maneira geral a $\mathrm{CT}$ pode ser considerada como o foco e o embasamento tecnológico da empresa, ou seja, o diferencial em relação aos concorrentes, devendo dessa forma ser preservada e protegida através de direitos de propriedade industrial. Já as BT não devem fazer parte do foco tecnológico principal da empresa, pois são oriundas de terceiros ou de domínio público, podendo no máximo ocorrer parceria com o fornecedor para ajustes de projeto, qualidade e especificações.

No caso do processo de produção as tecnologias utilizadas também podem ser caracterizadas como "tecnologias principais" e "tecnologias complementares", porém são de natureza múltipla, ou seja, todo o processo ou cada etapa do processo de produção poderá utilizar uma "core technology" e várias "bondaries technologies", embutidas em suas máquinas ("bens de capital") de fabricação, bem como em outros equipamentos utilizados no processo de produção do produto. Nesse campo, de caracterização mais complexa, também a CT deve ser desenvolvida pela empresa e protegida através de direitos de propriedade industrial, pois essa fase é fundamental para a competitividade da empresa no mercado. Enquanto a tecnologia embutida em um produto pode ser pesquisada e copiada, pois está no mercado e à vista de qualquer consumidor ou concorrente, o mesmo não ocorre com a tecnologia embutida no processo, que é interna à empresa, preliminarmente não acessível ao consumidor e à concorrência. Similarmente ao caso do produto, as BTs do processo são originárias de terceiros, envolvendo equipamentos de produção e de processo.

Esse modelo de caracterização das tecnologias de produto e de processo, na indústria de manufaturados, permite analisar vários aspectos correlacionados a essas tecnologias, como: gestão das tecnologias (CT e BT) a curto, médio e longo prazo; prospecção tecnológica; vulnerabilidade tecnológica; melhorias em produtos e processos; formação, adequação e manutenção de capabilidade tecnológica; relação das tecnologias de produto e processo com o meio ambiente e conservação de energia; influência do setor de bens de capital no processo de produção etc.

\section{GESTÃO DA TECNOLOGIA NAS EMPRESAS}

A gestão da tecnologia nas empresas vem sendo estudada por muitos pesquisadores no exterior e no Brasil, entre esses por exemplo os trabalhos de Silva; Plonski (1996), Silva (1999), Silva; Plonski (1999), Silva (2002a), e Silva (2002b), onde analisam desde aspectos estratégicos até operacionais, incluindo pesquisa de campo. O contexto dessa abordagem está nas tecnologias de produto e de processo de produção do setor de manufaturados.

Figura 04 - Matriz de Relacionamentos das Atividades na Organização

\begin{tabular}{|c|c|c|c|c|c|c|c|c|c|c|c|c|c|c|}
\hline \multicolumn{15}{|c|}{ Gerenciamento Elobal da Empresa } \\
\hline $\begin{array}{c}\text { Matriz de } \\
\text { Relacionamento } \\
\text { das Atividades } \\
\text { na Organizaçáo }\end{array}$ & 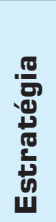 & 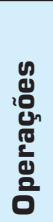 & 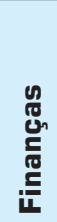 & 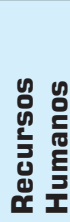 & 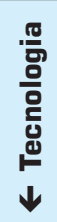 & $\begin{array}{l}\frac{0}{0} \\
\frac{\pi}{0} \\
\frac{\pi}{00} \\
0\end{array}$ & 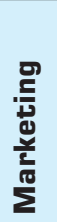 & 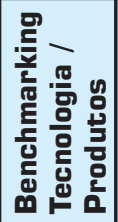 & 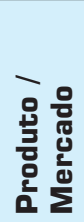 & 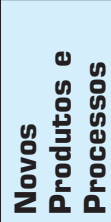 & 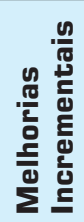 & 递蒂 & 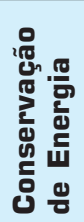 & 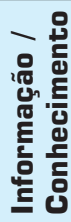 \\
\hline Estratégia & $\boldsymbol{*}$ & & & [ & [ & & $\mathbf{\square}$ & $\mathbf{\square}$ & [ & & & & & \\
\hline Operações & & $*$ & a & $\bar{a}$ & $\bar{\square}$ & $\square$ & & & & $\bar{\square}$ & $\mathbf{\square}$ & a & [ & $\square$ \\
\hline Finanças & & $\bar{\square}$ & 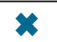 & $\bar{\square}$ & $\bar{\square}$ & & & & 口 & & $\square$ & & & $\bar{\square}$ \\
\hline Recursos Humanos & [ & [ & [ & $x$ & [ & $\mathbf{\square}$ & & & & & a & [ & $\mathbf{\square}$ & [ \\
\hline Tecnologia $\rightarrow$ & [ & $\bar{\square}$ & $\bar{\square}$ & $\bar{a}$ & $\bar{x}$ & $\mathbf{\square}$ & 口 & 口 & च & [ & $\mathbf{\square}$ & [ & [ & [ \\
\hline Qualidade & & 口 & & $\square$ & 口 & 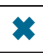 & 口 & $\square$ & [ & $\square$ & $\square$ & & & $\mathbf{\square}$ \\
\hline Marketing & $\bar{\square}$ & & & & $\bar{\square}$ & $\bar{\square}$ & $\boldsymbol{x}$ & $\overline{\mathbf{D}}$ & $\overline{\mathbf{a}}$ & $\bar{a}$ & $\bar{a}$ & $\bar{\square}$ & & $\bar{\square}$ \\
\hline $\begin{array}{l}\text { Benchmarking } \\
\text { Tecnologia/ Produtos }\end{array}$ & $\mathbf{\square}$ & & & & $\mathbf{\square}$ & घ & $\mathbf{\square}$ & $\boldsymbol{x}$ & [ & [ & & & & [ \\
\hline Produto / Mercado & [ & & [ & & [ & [ & [ & [ & * & [ & [ & & & [ \\
\hline Novos Produtos e Processos & & 口 & & & $\bar{\square}$ & $\square$ & $\square$ & $\square$ & $\square$ & 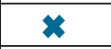 & & 口 & $\square$ & $\bar{\square}$ \\
\hline Melhorias Incrementais & & 口 & [ & [ & 口 & $\square$ & 口 & & 口 & & * & a & [ & a \\
\hline Meio Ambiente & & $\square$ & & $\square$ & $\square$ & & $\square$ & & & 口 & $\square$ & * & & $\square$ \\
\hline Conservação de Energia & & $\square$ & & $\square$ & $\square$ & & & & & 口 & $\square$ & & $*$ & $\square$ \\
\hline Informação / Conhecimento & 口 & $\bar{\square}$ & 口 & $\bar{\square}$ & $\bar{\square}$ & [ & 口 & 口 & [ & $\bar{\square}$ & $\square$ & [ & $\square$ & * \\
\hline
\end{tabular}


Embora a tecnologia seja uma dimensão de primeira ordem dentro de qualquer empresa, pois nenhum produto/ serviço poderá ser gerado sem sua utilização, ainda não é tradicional a existência de uma área específica, seja funcional ou virtual, dentro da maioria das organizações. Uma das razões que poderia ser colocada seria o sigilo, em função da vulnerabilidade tecnológica e da estratégia da empresa no mercado. Historicamente, a qualidade foi incorporada como área funcional, dentro das empresas, nas primeiras décadas do século XX. No campo da tecnologia, somente a partir da década de 1990 as empresas mais avançadas introduziram essa incorporação, porém de forma diferenciada, envolvendo mais efetivamente gerenciamento por processos do que a criação de uma área funcional. A Figura 4 esquematiza inter-relações da "dimensão tecnologia" com outras funções ou atividades da empresa.

Assim temos a "Matriz de Relacionamentos das Atividades na Organização" dentro do "Gerenciamento Global da Empresa", destacando-se a atividade "tecnologia". Como podemos constatar, a função tecnologia, juntamente com a função informação/conhecimento, se inter-relacionam com todas as outras funções da organização. A natureza dessas inter-relações, bem como a eficiência e a eficácia da função tecnologia, depende da forma organizacional da empresa. As organizações modernas, com sistemas consolidados de gestão da qualidade, têm maior facilidade para gerenciar essas inter-relações. Por outro lado, as organizações excessivamente funcionais, bem como aquelas que trabalham com "gerenciamento por objetivos", não dispõem de sistema de qualidade eficiente, e têm grandes dificuldades para gerenciar aquelas inter-relações. Essa constatação é decorrente do fato de que as organizações com sistema de gestão da qualidade moderno têm forma organizacional distinta daquelas com predomínio funcional. Desta forma, o aprendizado na implantação do sistema de gestão da qualidade poderá ser importante na implantação do sistema de gestão da tecnologia, sob o ponto de vista organizacional. Enquanto que no sistema de qualidade muitas atividades são repetitivas, em atendimento ao próprio fluxo da produção, no sistema de tecnologia as atividades são dinâmicas, pois todos os projetos de prospecção tecnológica e de desenvolvimento têm características individuais. Uma análise da Figura 4 fornece indicações da importância do "conceito da macrotecnologia" no contexto da capabilidade tecnológica.

Vamos agora detalhar alguns aspectos da gestão da tecnologia na empresa, a curto, médio e longo prazo, como pode ser observado na Figura 5.

Como podemos constatar, as "atividades correntes" envolvem o gerenciamento da utilização das tecnologias já desenvolvidas ou existentes na empresa, e todas as implicações dentro do sistema produtivo. O "desenvolvimento de futura capabilidade tecnológica" engloba atividades de prospecção tecnológica, de geração de novas tecnologias e de formação da própria capabilidade tecnológica da organização. As atividades "monitorando programas e melhoria

Figura 5: Inter-Relações de Aspectos da Gestão da Tecnologia na Empresa.

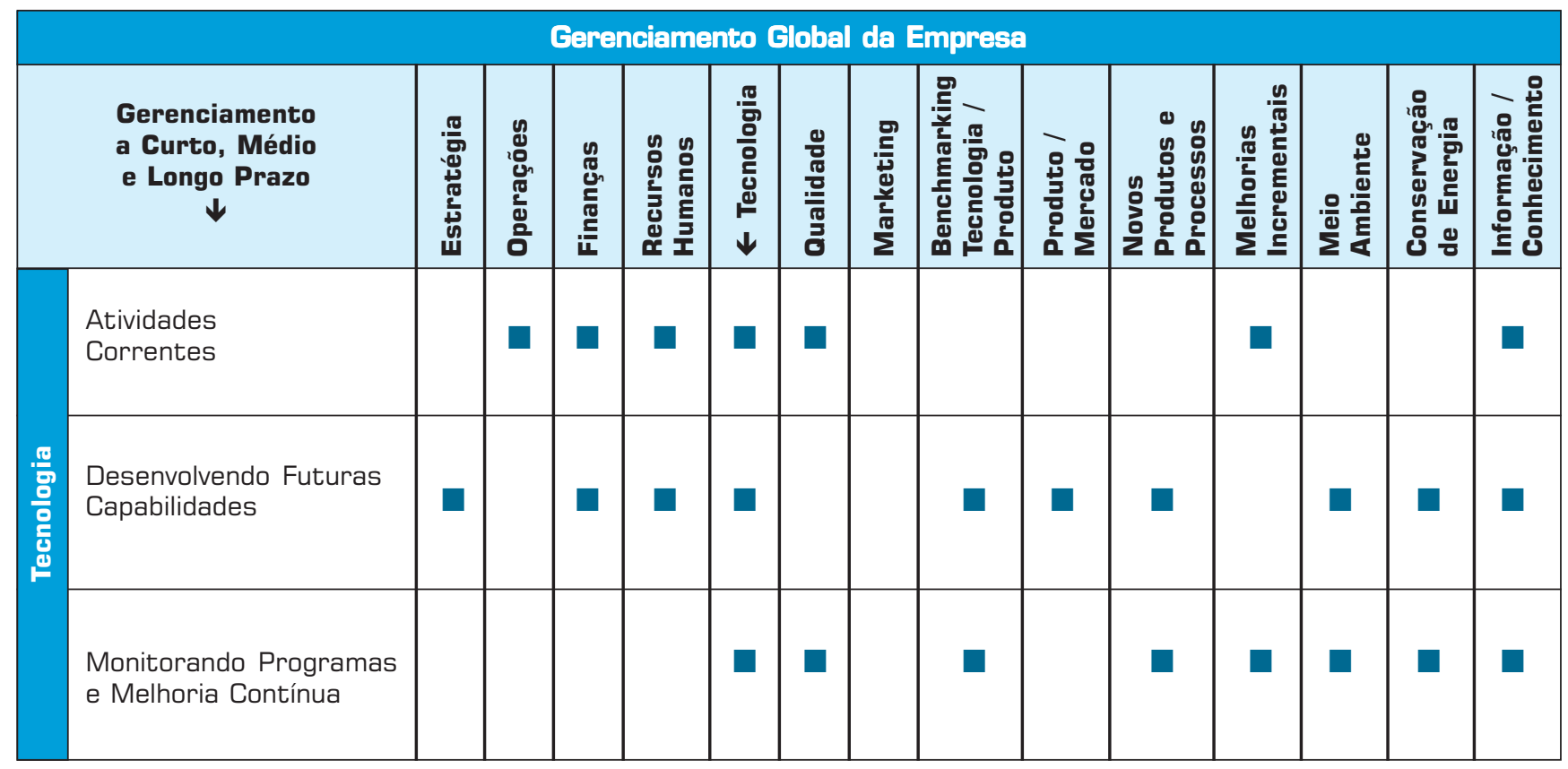


contínua" são geradoras da etapa anterior, e envolve aspectos estratégicos no campo da tecnologia e inter-relações com outras áreas da empresa, visando a melhoria contínua das tecnologias de produto e de processo. Pode-se dizer que as atividades especificadas em curto prazo estão enfocadas mais em desdobramentos da dimensão "microtecnologia", enquanto que as atividades de médio e longo prazo na dimensão "macrotecnologia".

\section{ANÁLISE DE CAMPO: MACROTECNOLOGIA E MICROTECNOLOGIA}

A dimensão tecnologia, dentro de uma organização, é o fator chave que leva a empresa a gerar produtos e serviços para a comunidade. Pode-se dizer que se constitui no "segredo" mais relevante, cujo conteúdo é o conhecimento "gerado e armazenado" pelas pessoas da organização, de caráter restritivo, e dentro de um mundo real. Uma empresa não pode existir sem ter a capacidade de manejar as tecnologias que geram seus produtos e serviços. As tecnologias patenteadas dão à empresa alguma segurança relativa no mercado durante um certo período de tempo, o qual na prática é indeterminado, pois tecnologias alternativas poderão aparecer nesse mercado.

Abordamos anteriormente o significado da palavra "tecnologia", segundo vários autores, e desenvolvemos os conceitos da "macrotecnologia" e da "microtecnologia", abordados pelas Figuras 1 e 2. O conceito da microtecnologia permite caracterizar de maneira mais precisa o valor agre- gado das tecnologias de produto e de processo, e desdobrar a tradicionalmente chamada "tecnologia" em "tecnologia principal" e "tecnologias complementares", embutidas em um produto ou processo. Já o conceito de macrotecnologia visualiza o aspecto sistêmico dos conhecimentos embutidos em uma tecnologia, dentro da organização, ou seja, as interrelações entre pessoas, equipamentos, programas, e áreas funcionais no contexto daquela tecnologia.

Essa configuração e desdobramento permitem à empresa uma auto-avaliação de seus conhecimentos das diferentes tecnologias envolvidas em seu produto ou processo de produção, cujos resultados poderão direcionar os esforços para formação de capabilidade tecnológica dentro da organização.

A pesquisa de campo foi conduzida no ano de 2001, através de entrevistas em 35 empresas, selecionadas dentre as 82 empresas utilizadas no trabalho desenvolvido por Silva; Plonski (1999), com análise de dados segundo Siegel (1975) no campo da estatística não paramétrica, utilizando o projeto de "pesquisa qualitativa" e o método de pesquisa "entrevista semi-estruturada", de acordo com a metodologia de Bryman (1989). No campo da microtecnologia, a Figura 6 apresenta a análise do "valor agregado tecnológico" e do "domínio das tecnologias principais" de produto e de processo no contexto das empresas. Com relação à tecnologia principal do produto, seu valor agregado é considerado alto por somente $6 \%$ das empresas, médio por $35 \%$, e baixo por $59 \%$. Esses valores estão compatíveis, tendo em vista

Figura 6: Microtecnologia: Valor Agregado e Domínio das Tecnologias.

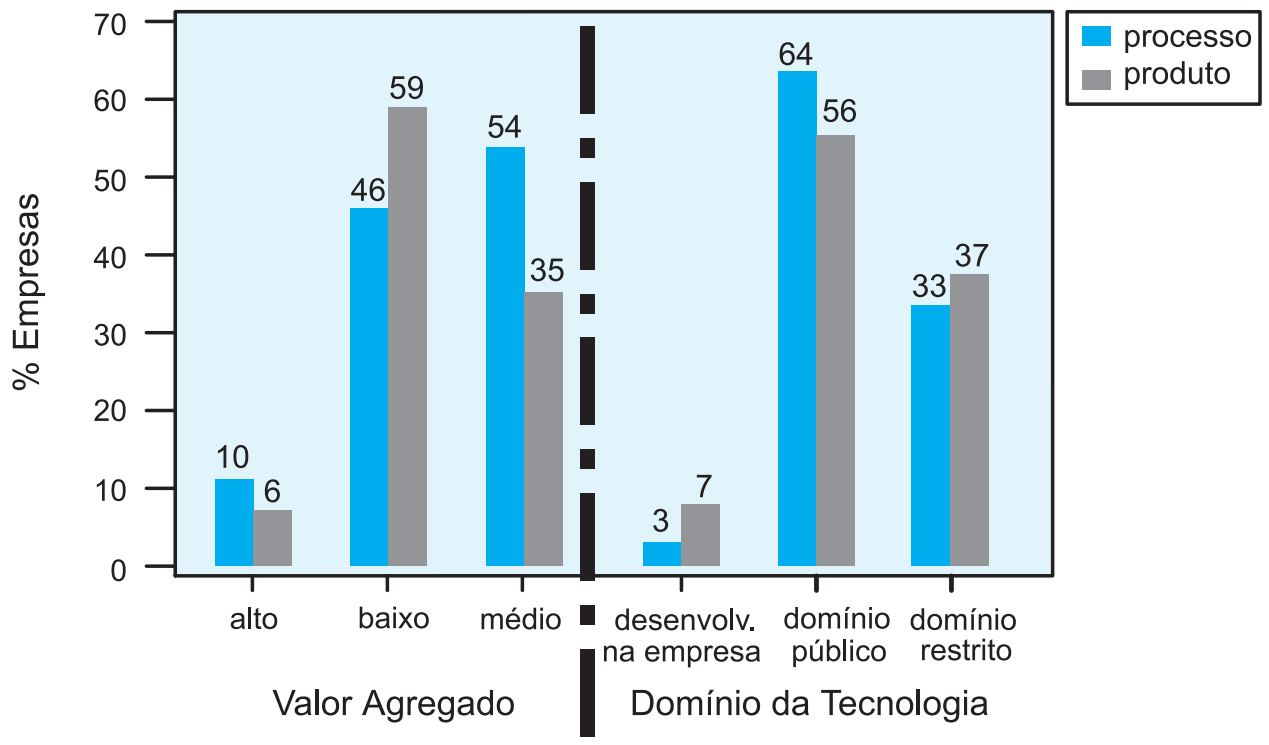


que a maioria das empresas pesquisadas pode ser considerada tradicional no setor de manufaturados, não envolvendo alta tecnologia. No caso da tecnologia principal de processo, o valor agregado é considerado alto por $10 \%$ das empresas, médio por $54 \%$, e baixo por $46 \%$, indicando que a maioria das empresas (64\%) considera seus processos de produção razoavelmente competitivos no mercado em que atuam.

Com relação ao "domínio das tecnologias" de produto, $7 \%$ das empresas indicam desenvolvimento próprio, $37 \%$ de domínio restrito, e 56\% de domínio público. Esses dados estão coerentes com aqueles delineados na análise do "survey" anterior, citados em Silva; Plonski (1999). Quanto ao "domínio das tecnologias" principais de processo, $3 \%$ das empresas indicaram desenvolvimento próprio, 33\% domínio restrito, e $64 \%$ domínio público. O conjunto desses dados indica dependência da maioria das empresas das tecnologias embutidas nos bens de capital. Por isso, o setor de bens de capital tem influência relevante nas tecnologias de processo e de operação nas empresas, merecendo ser estudado com mais detalhamento no campo da gestão da tecnologia.

A Figura 7 apresenta, ainda no campo da microtecnologia, dados sobre o domínio das tecnologias complementares de produto e processo nas empresas, utilizando uma escala que relaciona: utilização, conhecimento, controle, manejo, alteração. Com relação ao domínio sobre a tecnologia complementar de produto, apenas $26 \%$ das empresas indicaram sua utilização, $45 \%$ têm conhecimento, $24 \%$ controlam, $32 \%$ manejam, e somente $5 \%$ têm capacitação para alteração dessas tecnologias. No que se refere ao domínio das tecnologias complementares de processo, $100 \%$ das empresas fazem sua utilização, $32 \%$ têm conhecimento, $19 \%$ controlam, $25 \%$ manejam, e somente $2 \%$ têm capacidade de alterar essas tecnologias.

O conceito da "macrotecnologia", que envolve uma visão interfuncional sistêmica da tecnologia, incorpora informações, conhecimentos, estrutura e comportamento das pessoas, e se aplica a qualquer tipo de tecnologia utilizada dentro de uma organização. Assim, sua análise deve considerar diferentes aspectos, entre eles alguns itens pesquisados ao longo do presente trabalho, e outros que merecerão continuidade futura. Com base na análise efetuada anteriormente, através do "survey" e das entrevistas, quanto aos diferentes aspectos da gestão da tecnologia na empresa, podemos agora concluir preliminarmente que no contexto dos diferentes "componentes da macrotecnologia", as principais dificuldades das empresas pesquisadas estão concentradas em: "informação e conhecimento", "pessoas e comportamentos", "processos", e "organização". Como os outros componentes são decorrentes e parte inseparável do sistema, concluímos por limitações na macrotecnologia para a maioria das organizações consultadas.

Quanto maior o valor agregado das tecnologias embutidas em produtos ou processos, maior a capabilidade tecnológica da organização e possibilidade de competitividade no mercado. Por outro lado, quando o valor agregado é baixo, para todos os produtos e processos, a empresa não

Figura 7: Domínio das Tecnologias Complementares de Produto/Processo.

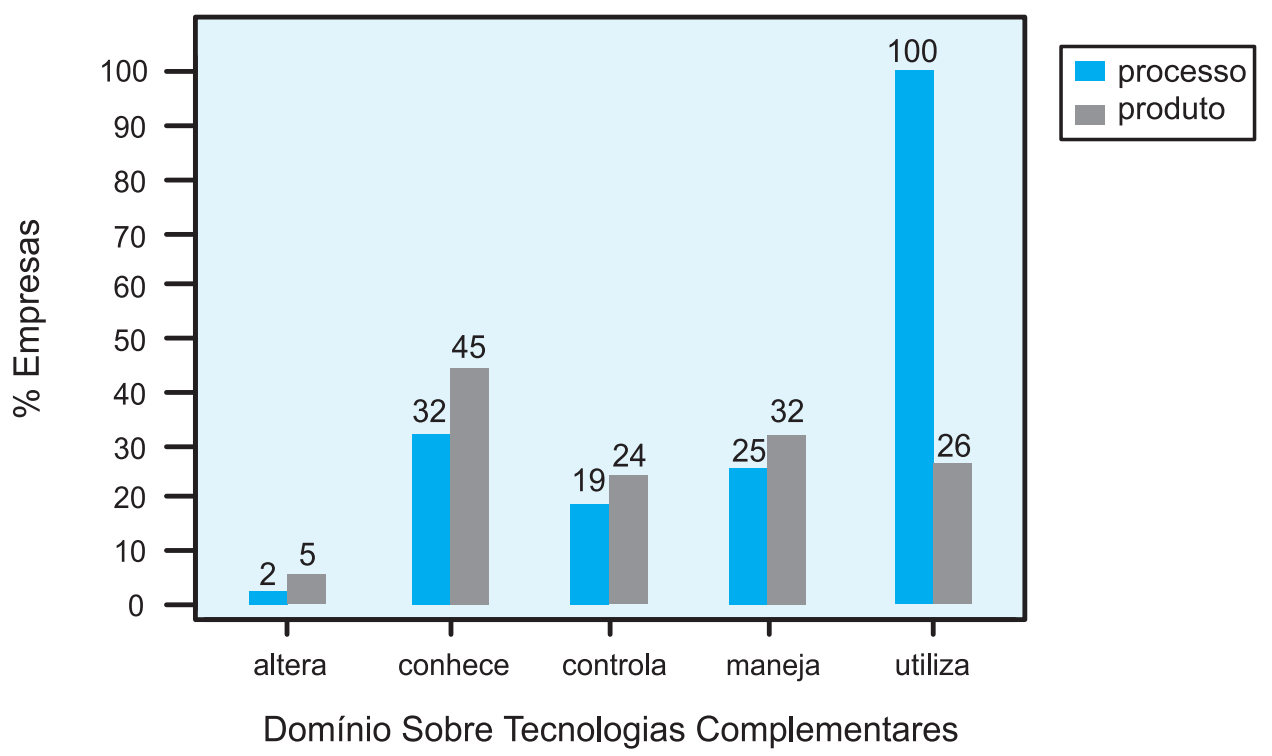


dispõe de capabilidade tecnológica e terá constantes dificuldades para competir no mercado. É necessário destacar que para uma grande parte de produtos manufaturados o valor agregado tecnológico não é substancial, ou seja, é de domínio público, o que teoricamente abre a possibilidade de qualquer empresa do ramo fabricar aqueles produtos. Nesse contexto tecnológico, a diferenciação de competitividade entre as empresas poderá estar na microtecnologia de processo, que seria nesse caso o fator chave dominante.

O nível de desenvolvimento de um país ou região está diretamente relacionado ao estágio científico e tecnológico de sua comunidade nos diferentes ramos do conhecimento. Dentro dessas comunidades existe uma configuração média de conhecimento tecnológico, que poderíamos chamar de "capabilidade tecnológica de domínio público", que é maior quanto mais desenvolvido for o país ou região. Esse conhecimento tecnológico difundido para as pessoas, no sentido coletivo, pode ser considerado como uma "aprendizagem da comunidade" ao longo do tempo, e constitui um fator importante para o desenvolvimento das empresas inseridas nesse meio. Paralelamente a esse "conhecimento médio tecnológico comunitário" estão as tecnologias patenteadas ou de domínio restrito, as quais não são difundidas para a comunidade durante um certo período de tempo, em função dos direitos de propriedade industrial e/ou da maturação do uso dessas tecnologias em bens e serviços. Após esse período essas tecnologias se difundem e se incorporam ao conhecimento tecnológico comunitário, inicialmente da região e depois do país. O conhecimento tecnológico é gerado pelas necessidades de uma determinada comunidade, sendo sua translação para outras comunidades um verdadeiro paradigma, podendo repercutir e influenciar aspectos sociais e culturais da sociedade receptora, positivamente ou negativamente. Como as comunidades têm também muitas características próprias, essas necessidades podem ser diferenciadas, com a conseqüente geração de tecnologias também diferenciadas. Essa dinâmica e diversificação são fatores importantes para o desenvolvimento científico e tecnológico dos povos, com evolução cíclica e diferenciação regional entre as nações

\section{CONCLUSÃO}

O presente trabalho, concentrado na linha de pesquisa "gestão da tecnologia" é continuidade da pesquisa organizacional que vem sendo desenvolvida durante os últimos 6(seis) anos, cujos resultados têm sido publicados nas prin- cipais revistas indexadas e congressos do País, no campo da engenharia de produção. Procurou dar continuidade e desenvolver novos conceitos importantes dentro da gestão da tecnologia nas empresas, enfocando as tecnologias de produto e de processo de produção. Os estudos realizados não contemplam o termo "tecnologia", como vem sendo atualmente utilizado, nas relações entre hardware e software, dentro da tecnologia da informação, pois essa terminologia é genérica e tem caráter mais popular do que científico. Os aspectos da informação e tecnologia da informação foram evidentemente considerados, dentro do presente estudo, no que eles realmente representam dentro do campo da gestão da tecnologia, em produtos e processos nas empresas.

\section{— m síntese, a microtecnologia é o resultado final da macrotecnologia, onde é gerada e construída a capabilidade tecnológica através das inter-relações entre os diferentes setores de uma organização e entre a organização e o meio exterior.}

A pesquisa procurou levantar os aspectos atuais mais importantes dentro do campo da gestão da tecnologia nas empresas, em nível internacional, bem como desenvolver novos conceitos de "tecnologia" do produto e de processo, como a "microtecnologia" e a "macrotecnologia". O modelo conceitual, através das dimensões estratégica e operacional da tecnologia, induziu ao desenvolvimento de amplo trabalho de campo, envolvendo originalmente o levantamento "survey" como projeto de pesquisa e o "questionário auto-administrado" como método de pesquisa, complementados posteriormente pelo projeto de "pesquisa qualitativa" e método de pesquisa "entrevistas semi-estruturadas", abrangendo 35 empresas de várias cidades do Estado de São Paulo e Capital, com razoável nível de industrialização.

Foram analisados e discutidos vários elementos da gestão da tecnologia na empresa, envolvendo aspectos gerais, estratégicos e operacionais da tecnologia, como também a configuração dos novos conceitos de microtecnologia e macrotecnologia, e finalmente a caracterização da tecnologia e da gestão da tecnologia dentro das relações interfuncionais nas organizações. Em síntese, podemos dizer que a maior parte das organizações consultadas ainda não despertou para a importância da tecnologia para a competitividade no mercado globalizado, diferentemente das empresas de países desenvolvidos, onde as ações efetivas nesse campo surgiram a partir da década de 1990. Ironicamente, 
muitas empresas ainda estão no mercado interno porque não ocorreu essa mobilização na maioria das organizações. Todavia, elas não têm condições de competitividade no mercado internacional de países desenvolvidos, onde se concentra o maior poder aquisitivo.

Alguns movimentos que ocorreram nos EUA no início da década de 1990, envolvendo os meios acadêmicos, governamental e empresarial, no campo da inovação tecnológica, estão tendo reflexos iniciais somente agora no Brasil no âmbito dos Governos Federal e Estadual, com a criação de programas e fundos de apoio ao desenvolvimento de novas tecnologias. No setor privado constatam-se algumas ações de associações e confederações, procurando conscientizar os empresários da importância do desenvolvimento tecnológico. Não basta a substituição de equipamentos por atualmente utilizadas, bem como programar o desenvolvimento e uso de novas tecnologias para aquele processo e operações, ou para novos processos e novas operações. A simples divisão do processo produtivo em micro processos, dentro de um modelo linear, poderá induzir a equívocos nessa análise.

Uma análise da organização sob o ponto de vista da microtecnologia permite delinear os resultados finais da capabilidade da empresa em dois campos importantes e distintos: tecnologia de produto e tecnologia de processo produtivo. O valor agregado da tecnologia de produto configura a empresa no contexto do mercado, pois seus produtos e serviços representam o espelho da empresa para a comunidade. Já as tecnologias de processo produtivo constituem um conjunto de conhecimentos acumulados que permitem gerar produtos e serviços, de forma competitiva ou não, dependendo da capabilidade tecnológica da empresa no contexto do mercado. Em síntese, a microtecnologia é o resultado final da macrotecnologia, onde é gerada e construída a capabilidade tecnológica através das interrelações entre os diferentes setores de uma organização e entre a organização e o meio exterior.

As operações de um processo

bens de capital importados, como tem ocorrido nos últimos anos, com a isenção de impostos de importação, pois esse procedimento não incorpora capabilidade tecnológica à empresa. A organização deve se capacitar para desenvolver continuamente novos produtos viáveis, e investir no desenvolvimento de novas tecnologias para produtos e processos. Essa capacitação, que denominamos de capabilidade tecnológica, envolve todos os setores da organização em atividades contínuas, similarmente com o que ocorre em outras áreas. Se a forma organizacional da empresa for funcional, existe necessidade de criação de um setor específico de tecnologia. Se não for funcional, há necessidade de desenvolvimento de um processo que incorpore as atividades tecnológicas da organização. Independentemente da forma organizacional da empresa, a função tecnologia, quer seja específica ou virtual, deve ser desenvolvida com a integração entre os diferentes setores e níveis hierárquicos da empresa.

$\mathrm{O}$ gerenciamento por processo permite distinguir a diferença entre processo e operação, ou seja, uma análise de um processo produtivo sob dois pontos de vista. Nesse contexto, o uso do conceito de microtecnologia, com a tecnologia principal e tecnologias complementares do processo produtivo, permite uma análise tecnológica importante no delineamento dos valores agregados e domínio das tecnologias produtivo são conduzidas com o auxílio de instrumentos, programas, equipamentos e máquinas de produção, geralmente fornecidas por outras empresas, caracterizadas como do setor de bens de capital. Esse setor é considerado como a indústria de base para a implantação de outras empresas, com seu estágio tecnológico influenciando toda a cadeia produtiva e o desenvolvimento de um país. Desta forma, sugerimos pesquisar como as empresas do setor de bens de capital utilizam e desenvolvem as tecnologias embutidas em seus equipamentos, no campo da gestão da tecnologia. Um trabalho de pesquisa preliminar poderia ser, por exemplo, "gestão da tecnologia no setor de bens de capital", com desdobramentos futuros para outros projetos específicos em áreas importantes como agroindústria, siderurgia, energia, papel e celulose, eletrônica, alimentos, telecomunicações, ferroviária, automobilística, e outras.

Qualquer sistema produtivo, nas funções manufatura, serviços, transporte e suprimento, gera resíduos direta ou indiretamente, com maior concentração na indústria de manufatura. Muitos produtos eliminam resíduos com o seu uso durante o período de sua vida útil, e se tornam resíduos após esse período. Desta forma, toda a cadeia do sistema produtivo influencia o meio ambiente, através de uma relação direta com a gestão da tecnologia nas empresas, desde o projeto do produto e microtecnologia do produto, até o 
projeto do processo produtivo e a microtecnologia deste processo. Adicionalmente temos também a correlação entre a microtecnologia do processo produtivo as tecnologias específicas de reciclagem e tratamento de resíduos, dentro do sistema de controle ambiental da organização. Em face da importância desse campo, sugerimos projeto de pesquisa que proceda à análise, através de conceitos e de pesquisa de campo, das relações entre a gestão da tecnologia na empresa e a gestão do meio ambiente.

Outro campo de pesquisa importante está relacionado ao uso e conservação de energia em sistemas produtivos, que também tem relacionamento com os aspectos ambientais, mas merece ser destacado e estudado com detalhamento. Adicionalmente, temos também a correlação entre a microtecnologia do processo produtivo e as tecnologias específicas de conservação de energia, dentro do sistema de racionalização do uso de energia da organização. Em face da importância desse campo, dentro do programa de racionamento de energia elétrica, nas condições atuais do País, sugerimos projeto de pesquisa que proceda à análise, através de conceitos e de pesquisa de campo, das relações entre a gestão da tecnologia na empresa e a conservação de energia no sistema produtivo.

Concluindo, o presente trabalho constatou que ainda há grande potencial, de otimização e pesquisa, a ser explorado pelas empresas e universidades, envolvendo associações, governos federal e estaduais, visando implementação da capabilidade tecnológica das organizações, em direção à competitividade no mercado interno e externo. Temos procurado divulgar os resultados das pesquisas e ressaltar a importância desse campo para as empresas, através de conferências em órgãos representativos empresariais, como CIESP/ FIESP regionais, universidades, simpósios, congressos, e seminários de trabalho.

\section{Artigo recebido em 30/09/2002 \\ Revisado em 27/11/2002 \\ Aprovado para publicação em 13/02/2003}

\section{- Bibliografia}

BRYMAN, A. Research methods and organization studies. Londres : Unwin Hyman, 1989.

DERTOUZOS, M. L.; LESTER, R. S; SOLOW, R. M. Made in America: Regaining the Productive Edge. HarperPerennial : New York, NY, 1989.

DUSSAUGE, P.; HART, S.; RAMANANTSOA, B. Strategic Technology Management. John Wiley \& Sons : Chichester, UK, 1992.

FLEURY, A. C. C. Organização do trabalho industrial: um confronto entre teoria e realidade. São Paulo, 1978. Tese (Doutorado), Escola Politécnica, Universidade de São Paulo.

KRUGLIANSKAS, I. Tornando a pequena e média empresa competitiva. São Paulo : Instituto de Estudos Gerenciais e Editora, 1996
LONGO, W. P. Tecnologia e soberania nacional. São Paulo : Ed. Nobel, 1984.

MARTINO, J. P. Technological Forecasting for Decision Making. 2. ed., NorthHolland, New York, NY, 1983.

MERLI, G. Comakership: a nova estratégia para o suprimento. Tradução de Gregório Bouer. Rio de Janeiro, Qualitymark Ed., 1994. Cap.1, p.1-36: A evolução estratégica entre clientes e fornecedores.

SIEGEL, S. Estatística não-paramétrica (para as ciências do comportamento). São Paulo : Ed. McGraw-Hill, 1975.

SILVA, J. C. T. Modelo interativo empresauniversidade no desenvolvimento de produtos. São Paulo, 1999. 163p. Tese (Doutorado) - Departamento de Engenharia de Produção, Escola Politécnica, Universidade de São Paulo.
SILVA, J. C.T.; PLONSKI,G.A. Gestão da tecnologia : desafios para pequenas e médias empresas. Revista Produção, Associação Brasileira de Engenharia de Produção - ABEPRO - v.9, n. 1, p. 2330, Out. /99, Rio de Janeiro - RJ. (ISSN 0103-6513).

SILVA, J.C.T.; PLONSKI, G.A. Inovação Tecnológica : Desafio Organizacional. Revista Produção, ABEPRO, v. 6, n.2, p. 183-93, dez./1996. (ISSN 0103-6513).

SILVA, J.C.T. Dimensões de competitividade para a empresa brasileira: informação e conhecimento, qualidade, tecnologia e meio ambiente. Revista Transinformação-PUC, Campinas, v. 13 , n. 2 , p. $81-92$, julho/dezembro2001. (ISSN 0103 - 3786).

SILVA, J. C. T. Tecnologia: Conceitos e Dimensões. In: XXII ENCONTRO NACIONAL DE ENGENHARIA DE PRODUÇÃO -ENEGEP E VII CONGRESSO INTERNACIONAL DE ENGENHARIA INDUSTRIAL, Pontifícia Universidade Católica do Paraná-PUC-PR, Curitiba PR, Anais em CD ROM, Outubro/2002a.
SILVA, J.C.T. Gestão da tecnologia em empresas de manufatura. In: Tópicos Emergentes em Engenharia de Produção, v 1/ José Paulo Alves Fusco (organizador)São Paulo: Editora Arte \& Ciência, 2002b, cap. 13 , p. 285-324.(ISBN 857473-091-2)

STEENSMA, H. K. Acquiring technological competencies through inter-organizational collaboration: na organizational learning perspective. Journal of Engineering and Technology Management, v. 12 p. 267-86, 1996.

UTTERBACK, J. M. Mastering the dynamics of innovation. Boston: Harvard Business School Press, 1994.

WHEELWRIGHT, S. C. ; CLARK, K. B. Revolutionizing product development. New York. The Free Press, 1992. 